\title{
COMPUTATIONAL DESIGN OF SYNTHETICALLY FEASIBLE COMPOUNDS
}

\author{
P. Polishchuk \\ Institute of Molecular and Translational Medicine, Faculty of Medicine and Dentistry, \\ Palacky University and University Hospital in Olomouc, \\ 77900, Czech Republic, Olomouc, Hnevotinska 5
}

DOI: 10.19163/MedChemRussia2021-2021-167

E-mail: pavlo.polishchuk@upol.cz

The chemical universe is extremely large ( $10^{33}$ compounds). This makes systematic enumeration of compounds and their virtual screening not very efficient. De novo design proposes a reasonable alternative - compounds are generated on-the-fly in order to fit to the given property constrains. Thus, chemical space is adaptively explored and new uncovered subspaces can be visited. The main issue of computationally generated compounds is their synthetic accessibility.

There are multiple approaches to compound generation were suggested so far but reaction-based approaches were the most successful in terms of synthetic feasibility of generated compounds. However, they limit the search space and are not flexible to be combined with different search strategies. The most traditional implementation is stepwise growing of a molecule using reaction rules and a library of building block until the molecule reaches a desired property value or given maximum complexity.

Fragment-based approaches provide greater flexibility to structural modifications (growing, mutation or linking of molecules) but suffer from difficulties to control synthetic feasibility of generated compounds. We developed and implemented the framework of chemically reasonable mutations (CReM) which makes structural changes taking into account chemical context of fragments.

This results in always chemically valid structures and greatly increases control over synthetic feasibility of generated compounds [1-2]. We will demonstrate advantages of the new approach over traditional reaction-based approaches and approaches using modern generative neural networks. The most frequent use cases will be discussed as well as future perspectives of de novo design and hit/lead optimization based on this methodology.

\section{References}

[1] P. Polishchuk, Journal of Cheminformatics 2020, 12, 28.

[2] P. Polishchuk, Journal of Chemical Information and Modeling 2020, 60, 6074-6080.

$$
-167-
$$

